\title{
Interface Concepts for Communicating Green Cyber-Physical Systems to Public
}

\author{
Ljubo Mercep $^{1}$, Gernot Spiegelberg ${ }^{2}$, Alois Knoll ${ }^{1}$ and Jakob Stoeck ${ }^{1}$ \\ ${ }^{I}$ Chair for Robotics and Embedded Systems, Technische Universität München, Boltzmannstraße 3, Garching, Germany \\ ${ }^{2}$ Institute for Advanced Study / Siemens AG, Technische Universität München, Lichtenbergstraße 2a, Garching, Germany
}

Keywords: Cyber-Physical Systems, Sensor Data Flow, Human-Machine Interface, Data Visualization, Web of Things, Social Awareness, Smart Grid, Electric Mobility

\begin{abstract}
Complex interactions of cyber-physical systems, which are necessary to implement high-level functionality, should stay invisible to the outside world and to the user. However, there are times where an intuitive presentation of the inner workings of such systems might positively influence the acceptance of new technologies and sustainable business models. This is especially the case with the so-called green cyberphysical systems, based upon renewable energies, intelligent energy management and new mobility paradigms. In this work, an integrated approach to communicating the benefits of such systems was deployed in our demonstrator, the Innotruck. The vehicle can act as a micro-smart-grid, with the ability to buy, sell, consume, store and produce electric energy on the internal (partly virtual) or external (real) energy market. With its recharge stations it also serves as a support truck for other plug-in hybrid or pure electric vehicles. These use-cases are handled through a series of interconnected presentation concepts, allowing an insight into the technical systems with varying degrees of complexity. With our work, we have shown how the benefits of electric mobility, augmented by smart grids, can be communicated to broader public.
\end{abstract}

\section{INTRODUCTION}

Information and communication technology might play a crucial role in spearheading electric mobility. New system architectures can reduce the complexity and costs involved with adding new functionality to vehicles and integrating them into wider cyberphysical systems (Buckl, 2011).

The business model fitting the customer expectations will have to generate additional value through the cross-domain interaction of previously separated systems. Such is the interaction between the energy, communication and transport systems, emerging through the smart-grid and electric mobility concepts. The power grid of the future is based on renewable resources and therefore inherently volatile. This instability can be offseted through virtual energy buffers in the form of electric vehicles, which act together on an interconnected energy market (Negeri, 2012).

One of the goals of the Innotruck project is to showcase the advantages of such cross-domain approches from the area of system architecture and intelligent energy management through intuitive demonstrators.

This work is organized as follows. In section 2 we give a brief overview of our demonstrator, the Innotruck. In section 3 we propose several interface concepts. In section 4 we evaluate the demonstrator impact on the public and industry. We finally conclude in section 6 .

\section{DEMONSTRATOR FEATURES}

In this section we shortly describe the main features of the Innotruck, shown in figure 1. The research group behind the vehicle, based at the Technische Universität München, is led by Prof. Dr. Gernot Spiegelberg. The main lines of research are being pursued by doctoral candidates Claudia Buitkamp (drivetrain and energy optimization), Ljubo Mercep (human-machine interface), and Hauke Stähle (system architecture).

The Innotruck is a serial plug-in hybrid-electric drive-by-wire truck. It enables implementing and testing new concepts in the field of system architecture, energy management, human-machine 
interfaces and driver assistance, serving as both an innovation and a presentation platform. The truck is divided into three segments: presentation area with five large displays, business lounge with an additional display and the cockpit equipped with automotive HMI.

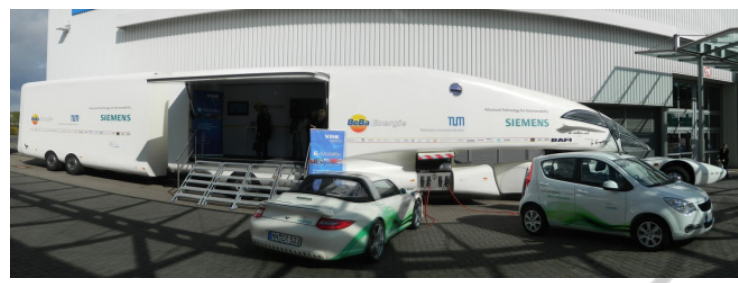

Figure 1: The Innotruck charging two electric vehicles.

With the Innotruck, we are able to present the underlying technology with various degrees of complexity. Depending on the previous knowledge and personal interest, the visitors can understand the vehicle as a charging station, an interactive exhibition stand for electric mobility, a drive-bywire hybrid truck and an active element of a larger smart grid. These aspects are shown in figure 2 .

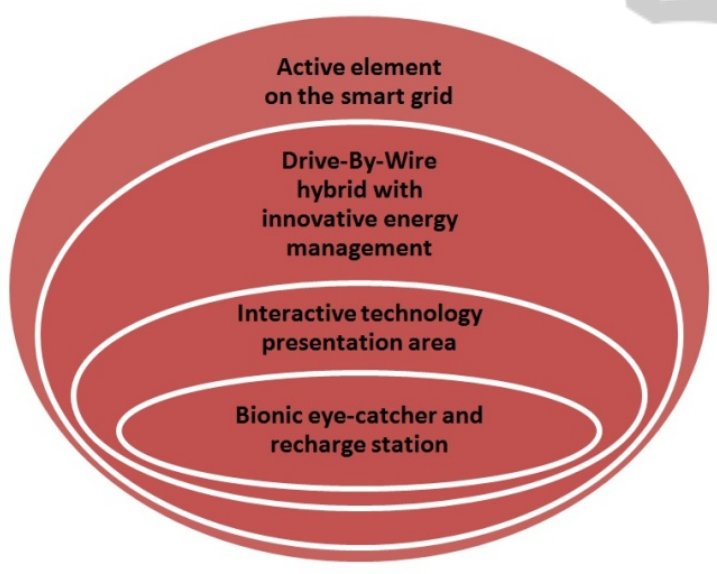

Figure 2: Various levels of vehicle perception.

In its current state, the vehicle is capable of driveby-wire operation over a sidestick-based input device (Daily Planet 3 November 2012). The second component of the automotive user interface is the integrated touchscreen console. It can be configured to run in a presentation mode when the vehicle is stationary, playing out simple driving scenarios.

\section{PROPOSED METHODS}

Several concepts for data presentation and interaction have been chosen. They all rely on the underlying sensor management framework, which has to satisfy following requirements:

- Plug-and-play capable

- Based on open standards

- Ontologies fitting any sensor system

- Easy integration in larger systems

- Easy integration with web-based services

- Possible extension into internet-of-things

After the analysis of the currently available approaches (Stoeck, 2012), the Sensor Web Enablement (SWE) information and service model from the Open Geospatial Consortium was chosen.

The information model describes the information being handled as well as the involved interfaces. The service model does the actual information handling and represents the actors in the data flow diagram (OGC, 2012). SWE overview is given in figure 3.

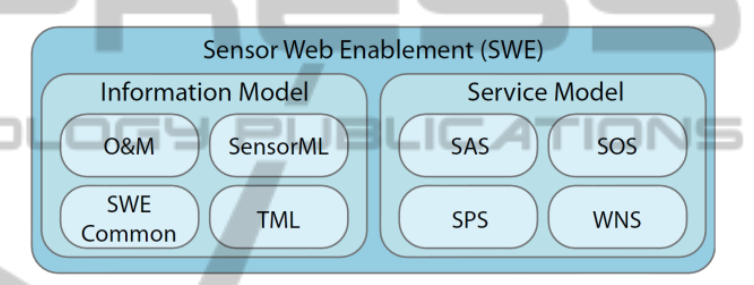

Figure 3: Main components of the SWE architecture.

\subsection{Machine-Machine Interfaces}

In order to enable in-vehicle communication between various subsystems as well as the communication to the outside world, we used the Sensor Observation Service Server (SOS) implemented by The 52 North Initiative in Java, running as a servlet in Apache Tomcat. The higher bandwidth usage of XML cannot be justified in the area of machine-machine interfaces, but enable a unified approach to sensor and observation description in human-readable format. The Efficient XML interchange (EXI) format proposed by the World Wide Web Consortium (W3C) in 2011 is one of the solutions to this issue (Kabisch, 2011).

\subsection{Human-Machine Interfaces}

There are two interface types corresponding to the two vehicle modes:

- Automotive HMI during the drive

- Presentation HMI used in stationary mode

We focus on the latter, further divided into three aspects: drive-by-wire hybrid truck, micro smart grid and charging station / fleet support, as shown in figure 4 . 


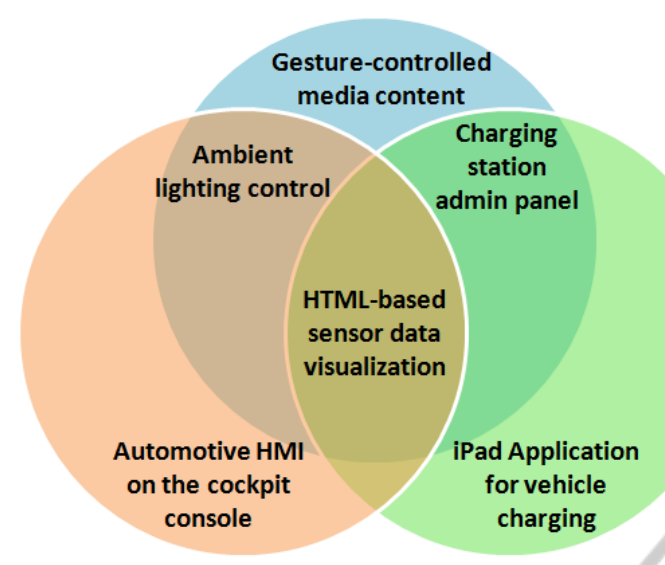

Drive-By-Wire hybrid truck

\section{Micro smart grid}

\section{Charging station / Fleet support}

Figure 4: Addressing vehicle aspects with different technical solutions. $\square$ AN

\subsubsection{Drive-by-Wire Hybrid Truck Aspect}

Main presentation element of this aspect is the simulation mode on the central driving console, as shown in figure 5 .

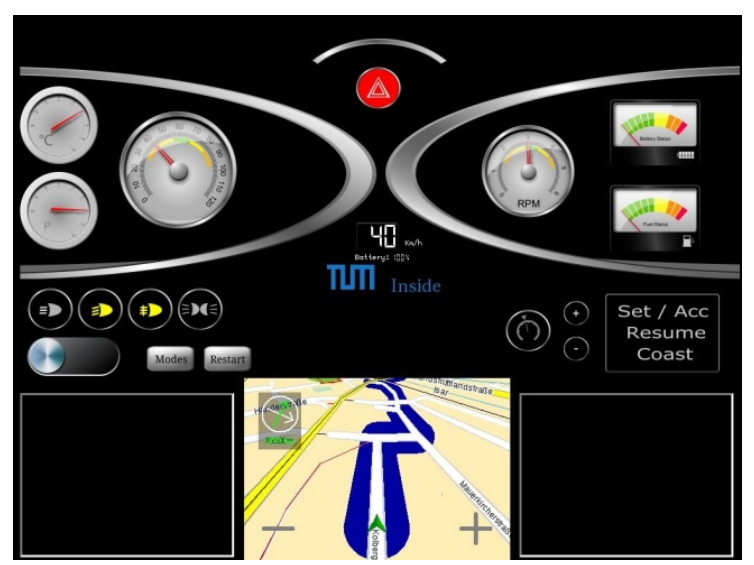

Figure 5: Driving simulation mode shown on the central vehicle console.

\subsubsection{Micro Smart Grid Aspect}

The energy, data and so-called financial flow is presented on one of the large displays in the presentation area, as shown in figure 6 .

Gesture control of the media content is realized with Microsoft Kinect-based solution and implemented on the displays in the presentation area

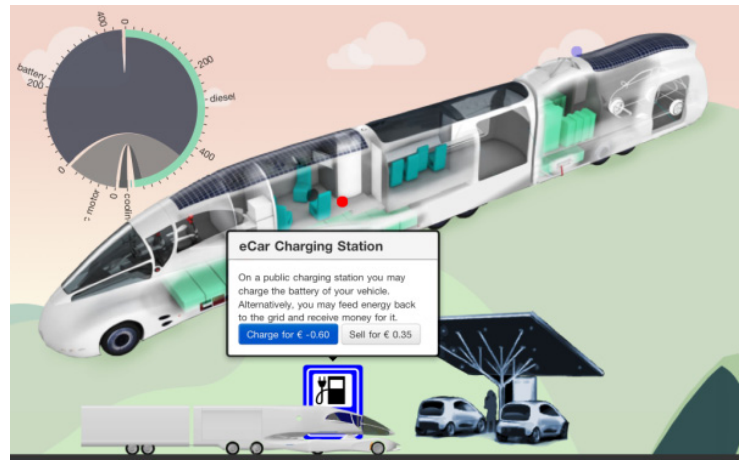

Figure 6: HTML-based sensor data visualization.

An ambient lighting control system has been installed, which can separately regulate ambient light intensity and colour in every Innotruck segment.

\subsubsection{Charging Station / Fleet Support Aspect}

The application managing / the vehicles/being charged at the Innotruck is iPad-native (Mercep, 2012). The iPad can connect ad-hoc to the embedded controller inside the vehicle or it can do so remotely over an internet connection. Some views of the userside of the applications are in figure 7. Admin panel has been developed in Nokia/digia QT.
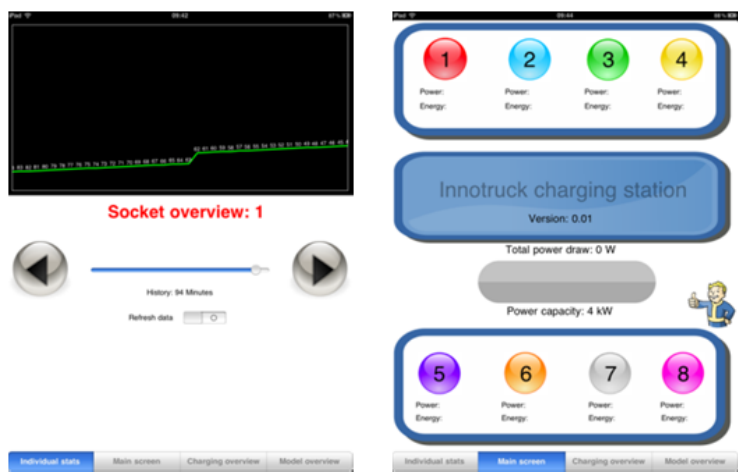

Figure 7: Application for vehicle charging management.

\section{PROJECT IMPACT}

\subsection{Industry Impact}

Building up a technology demonstrator of this scope requires, above all, an intrinsically motivated team. However, industry support in the form of providing prototype systems is essential for showcasing crossdomain approaches. More than thirty partners of all 
sizes and different business models have been involved in the development. The resulting "ecosystem" gives an example of how the initial research and development costs can be offset through the benefit of a common presentation platform.

\subsection{Societal Impact}

Excluding the press, the visitors can be divided into non-experts, industry representatives, enthusiasts and students. On the Hannover Messe 2011 and 2012, briefings were given to guided student groups, as shown in figure 8 .

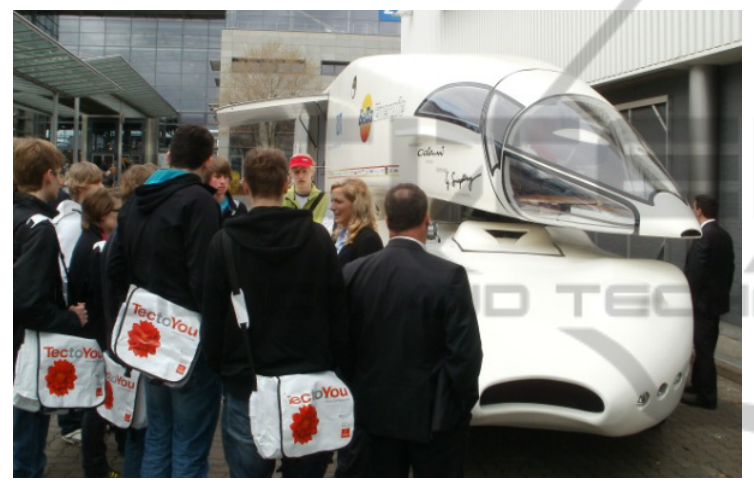

Figure 8: TecToYou student group being briefed at the truck.

The non-experts are mostly trying to familiarize themselves with new technologies through interactive media content in the presentation area, with a brief visit to the other vehicle segments. Industry representatives prefer personal briefings and presentations in the vehicle's business lounge. Enthusiasts and students prefer a more hands-on approach, trying to use every interaction point inside the demonstrator.

From the media relations point of view, the project is a definite success. More than 50 original articles and TV or video pieces have been found online or in printed form, excluding portals and websites which disseminate existing news articles.

\section{CONCLUSIONS}

We have demonstrated how intuitive interfaces on top of a data-centric sensor framework can effectively communicate science and technology involved in complex cyber-physical systems to broad public. This, in turn, creates ecosystems of tightly connected industry and academic partners, with common research and development interests. Such focus groups, working on the borders of different disciplines, bring out the added value stemming from the cross-domain interaction. With this vehicle we also present a cycle of knowledge generation, transfer and application, represented by the academic partners, translational research and application-oriented partners.

\section{ACKNOWLEDGEMENTS}

We would like to thank the International Graduate School of Science and Engineering (IGSSE), the Institute of Advanced Study (IAS) and the fortiss Institute of the Technische Universität München for all the help and practical support with the project.

\section{REFERENCES}

Buckl, C., Schätz, B., Fehling, M., Kuhn, K., Klein C. (2011). Mehr Software (im) Wagen: Informations- und Kommunikationstechnik (IKT) als Motor der Elektromobilität der Zukunft. Abschlussbericht des vom BMWi geförderten Verbundvorhabens "eCarIKT-Systemarchitektur für Elektromobilität".

Schaub, B. (Producer). (2012, November 3). The Innotruck test drive. In Daily Planet: Episode November 3rd-5th Part 2. Discovery Channel Canada.

Kabisch, S., Peintner, D., Heuer, J., Kosch, H. (2011). Optimized XML-based web service generation for service communication in restricted embedded environments. In IEEE Emerging Technologies \& Factory Automation (ETFA) 16th Conference, 1-8.

Mercep, Lj., Spiegelberg, G., and Knoll, A. (2012). The App for the charging management of „Innotruck“. In 6. VDI/VDE Fachtagung USEWARE 2012 - MenschMaschine-Interaktion. Deutsches Forschungszentrum für Künstliche Intelligenz, Kaiserslautern.

Negeri, E., Baken, N. (2012). Smart integration of electric vehicles in energy community. SMARTGREENS 2012 - $I^{\text {st }}$ International Conference on Smart Grids and Green IT Systems.

Open Geospatial Consortium. (n.d.). Sensor web enablement DWG. Retrieved October 1, 2012, from http://www.opengeospatial.org/projects/groups/sensor webdwg

Stoeck, J., Mercep, Lj., Spiegelberg, G. and Knoll, A. (2012). Platform-independent interface for the management of sensor-generated power and data flows in an automotive data-centric architecture. In 6. VDI/VDE Fachtagung USEWARE 2012 - MenschMaschine-Interaktion. Deutsches Forschungszentrum für Künstliche Intelligenz, Kaiserslautern. 